\title{
Numerical Study of Bottleneck Flow with Varying Corridor Width and Motivation Using a Speed-Based Model
}

\author{
Jonas Rzezonka ${ }^{1,2} \cdot$ Mohcine Chraibi $^{1} \cdot$ Armin Seyfried $^{1,3}$. Ben Hein ${ }^{3}$. \\ Andreas Schadschneider ${ }^{2}$ \\ ${ }^{1}$ Institute for Advanced Simulation, Forschungszentrum Jülich, Germany, \\ E-mail: j.rzezonka@fz-juelich.de \\ 2 Institute for Theoretical Physics, University of Cologne, Germany \\ 3 School of Architecture and Civil Engineering, University of Wuppertal, Germany
}

Received: 24 August 2021 / Last revision received: 15 November 2021 / Accepted: 22 November 2021

DOI: $10.17815 / C D .2021 .132$

\begin{abstract}
In this study a simple speed-based model is employed to simulate an experiment of pedestrian bottleneck flow. The experiment revealed that the density near the bottleneck is influenced by the motivation of the pedestrians and the corridor width. In narrow corridors, distinct lanes are formed for pedestrians with low motivation. These lanes can disappear when the pedestrians have a high motivation to reach their target. We show that a speed-based model is - despite its relative simplicity- capable to reproduce the observed phenomena to a high degree.
\end{abstract}

Keywords Pedestrians $\cdot$ bottleneck flow $\cdot$ speed based model $\cdot$ motivation

\section{Introduction}

A large variety of models for pedestrian dynamics of different sophistication has been proposed. The focus is mainly on microscopic models ranging from cellular automata discrete in time and space to models governed by continuous second or first order differential equations [1]. Pedestrians are represented as two-dimensional objects called agents (usually represented by discs or ellipses, or cells in cellular automata) that interact with each other and the environment. The models are capable of capturing a multitude of self-organisation phenomena observed in pedestrian dynamics, like lane formation, stopand-go waves, clogging or intermittent flow at bottlenecks [2-5]. 
One important property common to all models of pedestrian dynamics is the concept of volume exclusion, i.e. two agents can not inhibit the same space at the same time. This property can easily be included in cellular automata models by limiting the maximal number of particles in a cell (usually to 1) [6]. In the other popular class of models this is usually not so easy. In force-based models the dynamics of each agent is governed by Newtonian physics. Pedestrians are described by self-driven particles with inertia. Volume exclusion in these models is often soft, realised by a repulsion force [7]. This can lead to overlapping of agents especially in high density situations and to intrinsic numerical instabilities even for small discretisation steps and unrealistic behaviour like oscillation of the agents [8-12]. In the following we use a variation of the speed-based model introduced by Tordeux et al. [13]. In this model volume exclusion is realized by a non-collision property enabling an instantaneous velocity change of the agents. This is similar to the mechanism in some cellular automata based traffic models. Since the model has been shown to successfully reproduce emergent phenomena like lane formation in bi-directional flow and counter flow at bottlenecks it is a suitable candidate to study microscopic interactions in crowds.

Bottleneck flow is one of the central problems in pedestrian dynamics and has been studied extensively both experimentally [6, 14-25] and theoretically [13,26-34]. Here an important factor is the motivation of the individuals to reach their target. Especially in emergency situations, e.g. evacuation of a crowded building because of imminent danger, pedestrians are highly motivated to exit the building as fast as possible while individuals in a large crowd entering a lecture hall in the early morning to attend a boring seminar are less motivated to be one of the first ones to enter. This impacts the dynamics of the system and changes the behaviour of the individuals as they might start to push their neighbours to gain an advantage to reach their target quicker [17, 22, 24, 33]. This behaviour can lead to new emergent phenomena, e.g. collective transversal motion of the crowd observed in experiments conducted by Garcimartín et al. in Navarra in 2018 [20] or the faster-is-slower effect observed in simulations [3,35]. This effect is still ambiguous in experimental studies [17,22-24]. In theoretical models, high motivation (e.g. initiated by a competitive situation) can be implemented in different ways. In the social-force model it is related to the desired velocity which increases for higher motivation [3]. In the floor-field model (a cellular automata based model), friction is introduced to describe the competition between individuals [4]. Highly motivated and competitive acting agents can inhibit each other from traversing to empty cells due the desire to enter the same cell in the same time-step. The implementation of motivation in our model is discussed in the next section.

We test the model in a bottleneck scenario studied in the experiments in [17]. The goal of this study was to determine whether the rules of conduct between pedestrians (e. g. social norms) can be influenced by geometrical restrictions near the bottleneck, especially by varying the corridor width leading to a bottleneck. Variations of the experiment were performed by changing the motivation of the participants (low vs. high motivation). This was achieved by letting the participants imagine that they want to enter a concert hall. For low motivation they should imagine that there are enough spaces with a good view, while for high motivation they were told that they want to be near the stage where space 
is rather limited. Multiple interesting phenomena were observed. It was found that both parameters have an influence on the density near the bottleneck. This density increases with increasing width of the corridor even though pedestrians have more space to distribute themselves in a wider corridor. When going from low to high motivation, there is also an increase in densities for all widths. For low motivation and narrower corridors, distinct lanes form in the corridor which can disappear in the high motivated scenario, or by widening the bottleneck. A numerical and analytical study was conducted on the phenomena using a cellular automata model [26] where the effects of the increasing density are hinted at. However, through the limited spatial resolution cellular automata models cannot capture all details. In this study we recreate the situation in a simulation to show that a simple first order model is capable of reproducing the emergent effects observed in the experiments.

\section{Model}

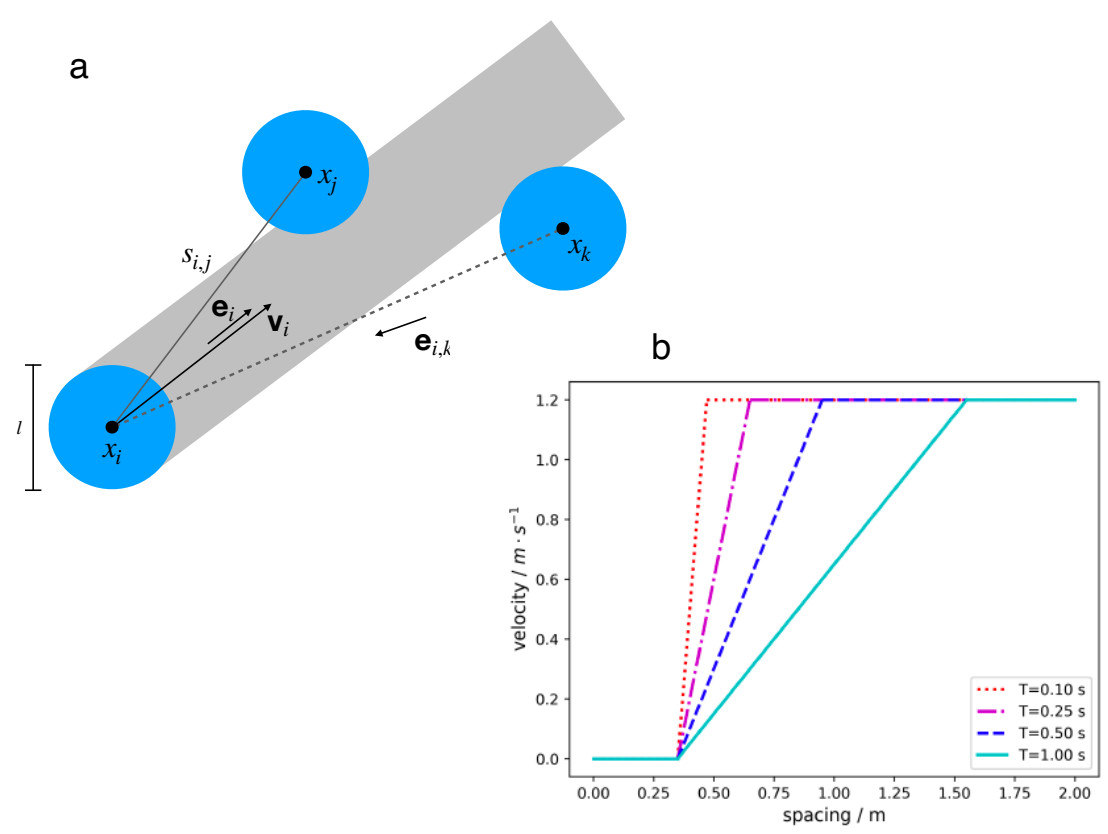

Figure 1 Illustration of the determination of speed and direction in the speed-based model. a: Definition of headway (grey area) and spacing $s_{i, j}$. b: Example of the optimal-velocity function for different slope factors $T$. The parameters used in the simulations are given in Table 1 .

The main idea of this study is to use a basic model with simple agent interactions to reproduce the empirically observed phenomena. The model utilised is a variation of the collision-free speed model introduced by Tordeux et al. [13] which will be explained later in the text. Agents in the model are represented by discs and adjust their speed to the nearest neighbour in their headway (i.e. the grey area in Fig. 1 a). The speed of agent $i$ is 
determined by the optimal-velocity function

$$
V\left(s_{i}\right)=\min \left\{v_{0}, \max \left\{0,\left(s_{i}-l\right) / T\right\}\right\},
$$

with the euclidean distance to the nearest agent in the headway $s_{i}$, the agent diameter $l$, the desired velocity $v_{0}$ and the slope factor $T$. The optimal-velocity function increases linearly with slope $T$ for headways $l \leq s_{i} \leq l+v_{0} T$ as illustrated in Fig. $1 \mathrm{~b}$.

The desired direction $\hat{e}_{i}$ is determined by a floor field through the Eikonal equation [36]

$$
|\nabla c(\vec{x})|=F(\vec{x}), \quad \vec{x} \in \Omega,\left.\quad c\right|_{\delta \Omega}=0,
$$

which calculates the quickest way to the target domain $\delta \Omega$ for an agent in the spatial

a

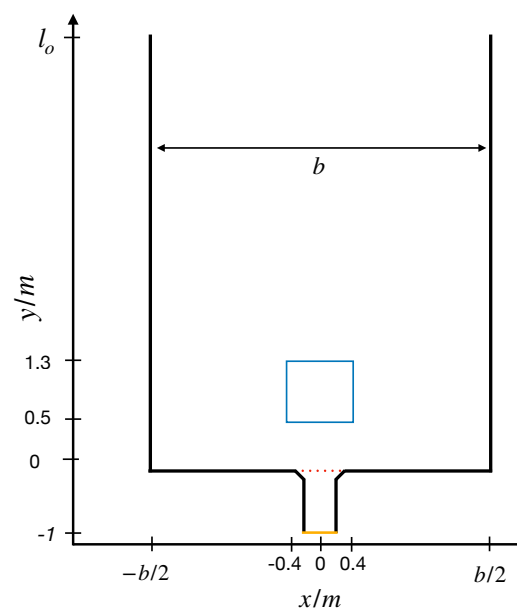

b

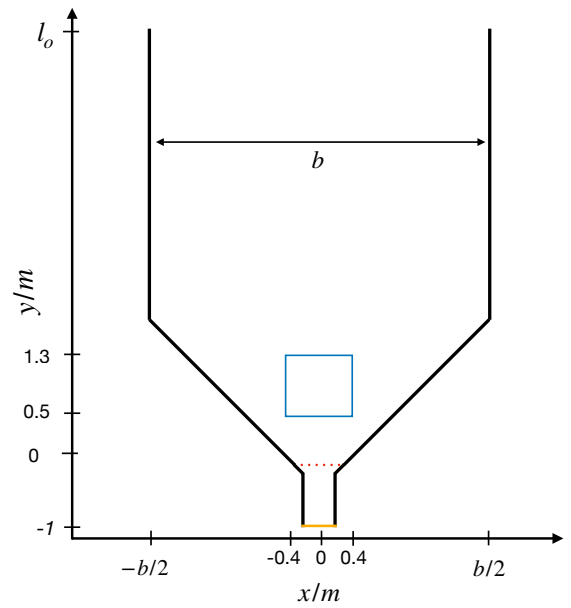

Figure 2 Illustration of the geometries used in the simulation. a: Geometry for high motivation with right angle configuration. b: Geometry for low motivation with a $45^{\circ}$ hopper shape simulating the effect of the railing on the floor. The measurement area is illustrated by the blue square. The center of the red dotted line $(\vec{r}=\overrightarrow{0})$ is used to measure the distance from the bottleneck.

domain $\Omega$. In this study the spatial domain is defined by the geometry of the corridor and bottleneck shown in Fig. 2 with the target domain $\delta \Omega$ being the orange line between $\mathbf{r}_{\mathbf{0}}=(-0.25,-1)$ and $\mathbf{r}_{\mathbf{1}}=(0.25,-1)$. The function $F(\vec{x})$ is called slowness field and determines the speed of a particle in the floor field with $v=1 / F(\vec{x})$ and $c(\vec{x})$ is the timecost for a particle to the target domain. This is used for the interactions with the wall as they are the source of a linear decreasing slowness field which deters the agents away from the wall [37]. The field is determined by the wall avoidance distance $d_{w}$ which is the point where the slowness field becomes zero. The variation to the model by Tordeux et al. [13] is that the exponential repulsion exerted by every agent on every other agent is replaced by a white-noise vector which is added to the desired direction $\hat{e}_{0}$. The $x$ and $y$ 
Table 1 Summary of model parameters and their values used in the simulations.

\begin{tabular}{lll}
\hline Parameter & Variable & Value \\
\hline Slope factor (motivation) & $T$ & $\{0.1,1.3\} \mathrm{s}$ \\
Desired velocity & $v_{0}$ & $1.34 \mathrm{~m} / \mathrm{s}$ \\
Agent size (hardcore exclusion) & $l$ & $0.35 \mathrm{~m}$ \\
Noise variance & $\sigma^{2}$ & 0.7 \\
Population & $N$ & 55 \\
Floor field resolution & $\Delta h$ & $0.01 \mathrm{~m}$ \\
Wall avoidance distance & $d_{w}$ & $0.34 \mathrm{~m}$ \\
Corridor width & $b$ & {$[1.2,5.6] \mathrm{m}$} \\
\hline
\end{tabular}

components of the vector $\vec{\zeta}$ are i.i.d. random numbers drawn from a normal distribution with zero mean and variance $\sigma^{2}$ :

$$
\hat{e}_{i}=\frac{\hat{e}_{0}+\vec{\zeta}}{Z}
$$

The factor $Z$ normalises $\hat{e}_{i}$. The additive noise helps to resolve gridlock situations between agents. With this the velocity $\vec{v}_{i}$ of agent $i$ is given by

$$
\vec{v}_{i}=V\left(s_{i}\left(x_{i}, x_{j}, \ldots\right)\right) \cdot \hat{e}_{i} .
$$

Because of the noise it would be possible for pedestrians to leave the geometry $\Omega$. If an agent would take a step outside of the geometry, this step is not allowed and the agent remains at its position.

In the model, different motivations are represented by varying the slope factor $T$. The idea is to find two values for $T$ which can reproduce the difference in density and lane formation observed in the experiment [17]. Highly motivated agents are simulated with a slope factor $T=0.1 \mathrm{~s}^{-1}$ while agents with low motivation with $T=1.3 \mathrm{~s}^{-1}$. Then pedestrians with low motivation will adjust their speed at larger distances to maintain more space between them and their neighbours while highly motivated pedestrians move with their desired velocity and fill gaps quickly. The number of agents is set to $N=55$ which is close to the average number of participants in the experiments (see next section). The desired velocity is set to $v_{0}=1.34 \mathrm{~m} / \mathrm{s}$, which is the mean walking speed measured for unobstructed pedestrians [38]. The agents size is chosen to be $l=0.35 \mathrm{~m}$. The parameters used in the simulation are summarized in Table 1.

\section{Experiments}

The experiments conducted by Adrian et. al [17] consisted of multiple runs for different corridor widths $b$ with a varying number of participants $N$ between 20 and 75 . In this study we omit the runs with $N<42$ to reduce finite size effects and have a smaller range in the participant number while still maintaining at least one run per corridor width. In the experiments a metal grating was partially covering the floor at the barriers which could 
have influenced the participants as they try to avoid stepping on it. This is visible in Fig. 3 (b) where the participants create a wedge shape in front of the bottleneck in the low motivation run avoiding to step on the metal grating. This effect disappears in the run with high motivation (Fig. 3 a). This can be observed clearly in the videos of the experiment [39]. To account for this observation in the simulations, two geometries are used as shown in Fig. 2. The geometry for low motivation runs is depicted on the right and has a hard wall in an $45^{\circ}$ angle leading to the exit while the geometry for high motivation has a $90^{\circ}$ angle to the bottleneck. The measurement area of the density is analogous to the experiments [17] and marked by the blue square in Fig. 2. The density is calculated using the Voronoi method [40]. The trajectory and video data of the experiment is available online [39].

C

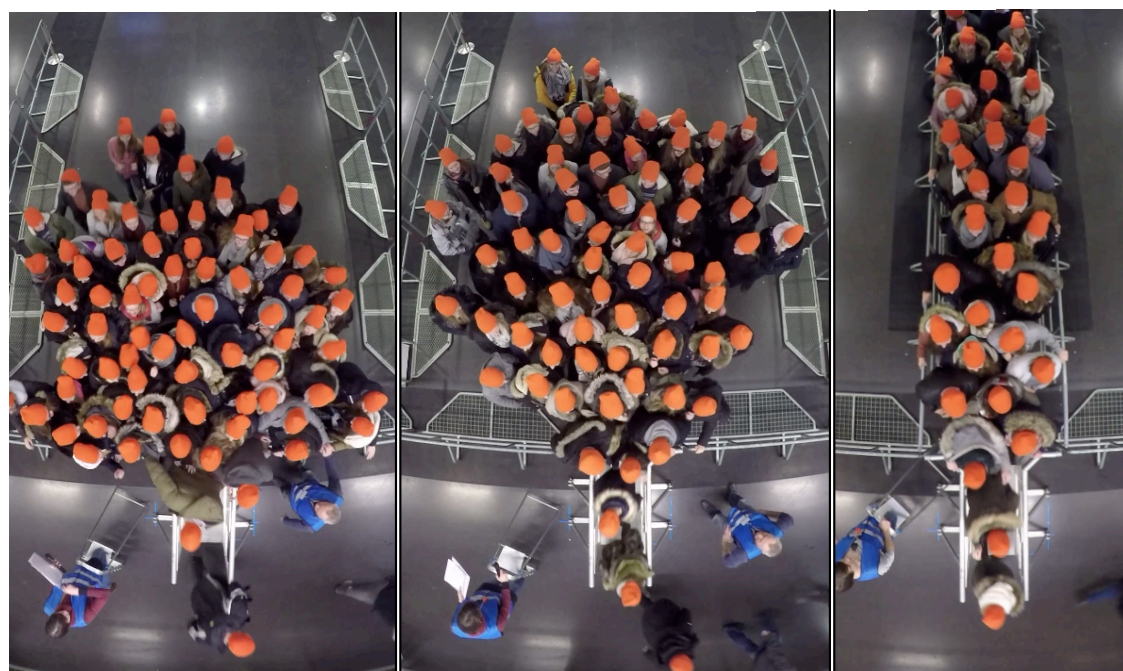

Figure 3 Snapshots from the experiment [17] with a: $b=5.6 \mathrm{~m}$ and high motivation; b: $b=5.6 \mathrm{~m}$ and low motivation; c: $b=1.2 \mathrm{~m}$ and high motivation.

\section{Results}

The experimental study mainly investigated two observable, the density near the bottleneck measured in the area defined in Fig. 2 and the time a pedestrian takes to get to their target $T_{t}$ (in this case the exit at $\vec{r}=\overrightarrow{0}$ ) in respect to the euclidean distance to the target $r_{t}$. In this study we compare these measures to the simulated model and additionally investigate the density structure of the experiments and simulations in the corridor.

Fig. 4 shows the time series of the experimental densities in the left column and the simulated densities in the right column. The top row shows the experimental data for high motivation runs while the bottom shows those for low motivation. As the number of participants varies, a large variation in the duration of the experiment can be observed. Comparing the density curves in the time frame from about $5 \mathrm{~s}$ to $20 \mathrm{~s}$ shows strong sim- 

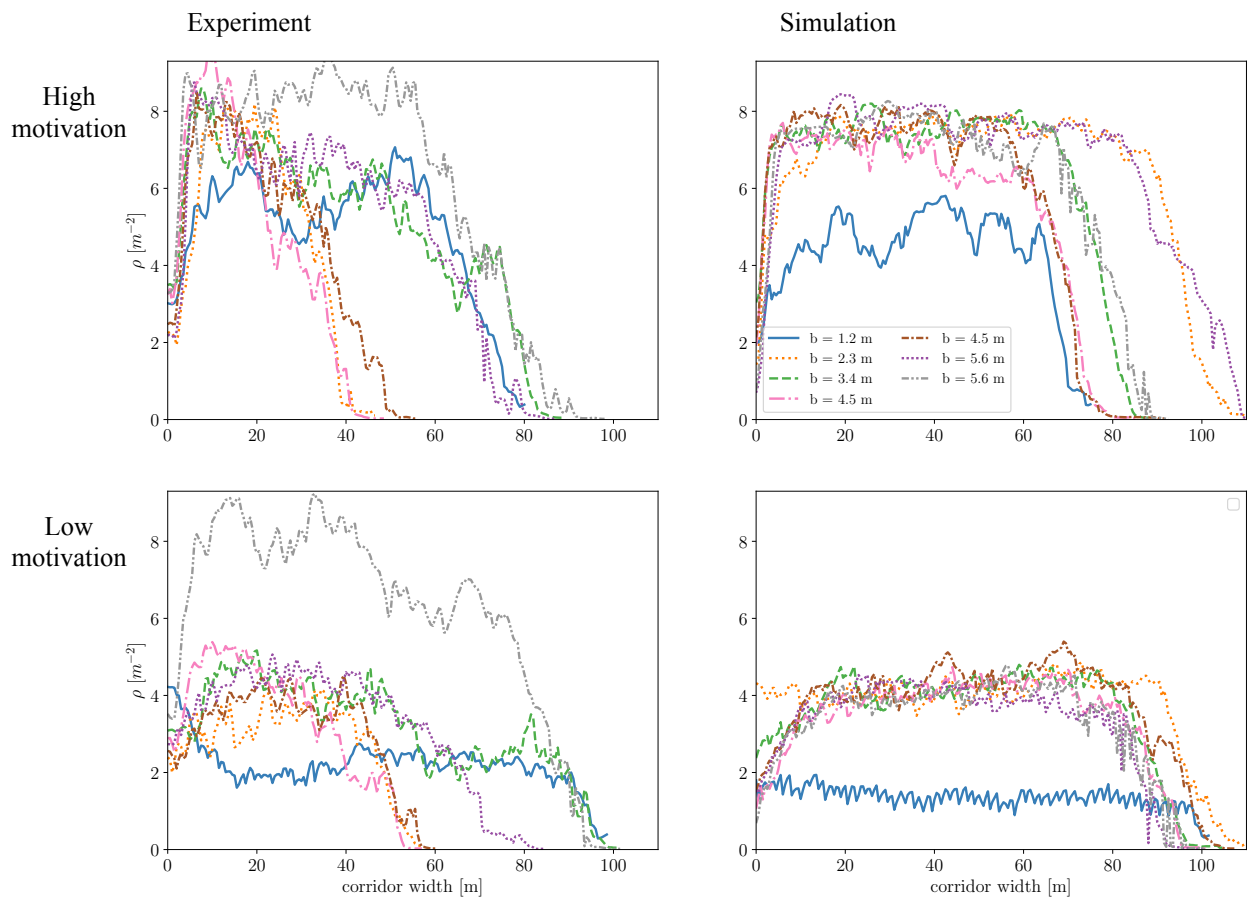

Figure 4 Time series of the density in the measurement area (see Fig. 2) for high motivation (top) and low motivation (bottom). On the left are the experimental runs and on the right the simulations. The parameters used in the simulations are given in Table 1.

ilarities between the simulated and experimental data. In both cases the density near the bottleneck increases with corridor width until it saturates at around $\rho \approx 4 \mathrm{~m}^{-2}$ for low motivation and $\rho \approx 8 \mathrm{~m}^{-2}$ for high motivation. In one of the $b=5.6 \mathrm{~m}$ runs for low motivation in the experiment the density is as high as in the high motivation run. This run has the largest number of participants with $N=75$, which could influence the dynamics together with the higher initial density of the participants. The experimental density profile is noisier than the profile of the simulations. This most likely stems from the inaccuracies in measuring experimental trajectories due to body and head movements, since the position of the head is measured. The density profile of the dynamics for different corridor width $b$ is depicted in Fig. 5. The density at any point $\vec{r}$ is defined as $\rho(\vec{r})=\sum_{i=0}^{N} \delta_{a}\left(\vec{r}-\vec{r}_{i}\right)$, where the delta-function is approximated by a Gaussian $\delta_{a}(x)=\frac{1}{\sqrt{\pi a}} \exp \left(-x^{2} / a^{2}\right)$. The parameter $a$ determines the width of the Gaussian. Its value should be chosen so that the Gaussian is wide enough to represent an agent with size $l$ but does not overlap too much with adjacent agents. In this study, it is set to $a=0.09$. With this value $\delta_{a}(l / 2) \approx 0.04$ and the full width at half maximum $F W H M=0.15$. This makes it possible to illustrate lane formation in the system. The density is measured from $10 \mathrm{~s}$ to $50 \mathrm{~s}$. From left to right the experimental and simulation data are shown in alternating order. The left two columns show high motivation while the right two show low motivation. Especially for low motivation distinct lanes are observed both in the experimental data and the simula- 
tion. For high motivation and $b=1.2 \mathrm{~m}$ the higher density configuration with more than two lanes emerges in the simulation by only changing the slope factor $T$. The wedge shaped geometry for low motivation in the experiment (Fig. 2 b) reproduces the density structure of the experiment very well supporting the assumption that the participants see the metal gratings as an obstacle. Looking at the relationship between the time to target or waiting time $T_{t}$ in respect to the distance $r_{t}$ in Fig. 6. the simulation fits fairly well to the experimental data, especially in the case of high motivation.

The regime of fast contraction (indicated by lines almost parallel to the $\mathrm{x}$-axis showing that individuals cover a large distance in a short time) in the first $4-5 \mathrm{~s}$ fits closely to the empirical results. The waiting regime in the low motivation case (indicated by lines almost parallel to the $y$-axis indicating that individuals almost stand still) is less pronounced in the simulations but can still be observed especially in the $b=1.2 \mathrm{~m}$ corridor. After the initial contraction phase, the regime of congestion begins where the individuals are closely packed together and in a jammed state. For low motivation, the simulations show a similar relationship between $T_{t}$ and $r_{t}$, going from almost linear for $b=1.2 \mathrm{~m}$ to a non-linear relationship as the corridor widens.
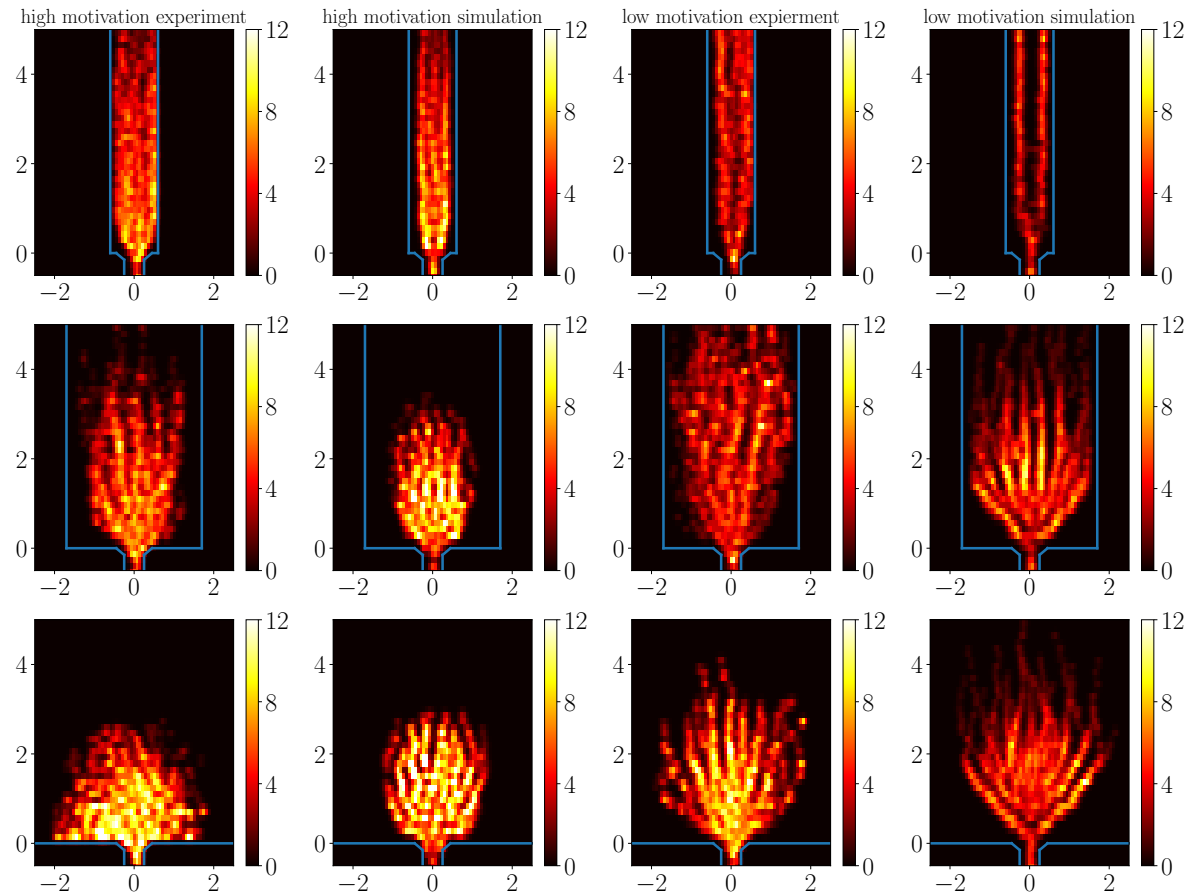

Figure 5 Density map of the experimental data and simulation data for $b=\{1.2,3.4,5.6\} \mathrm{m}$ from top to bottom. The experimental and simulation data is shown in alternating order, starting with the experimental data with high motivation on the left. 

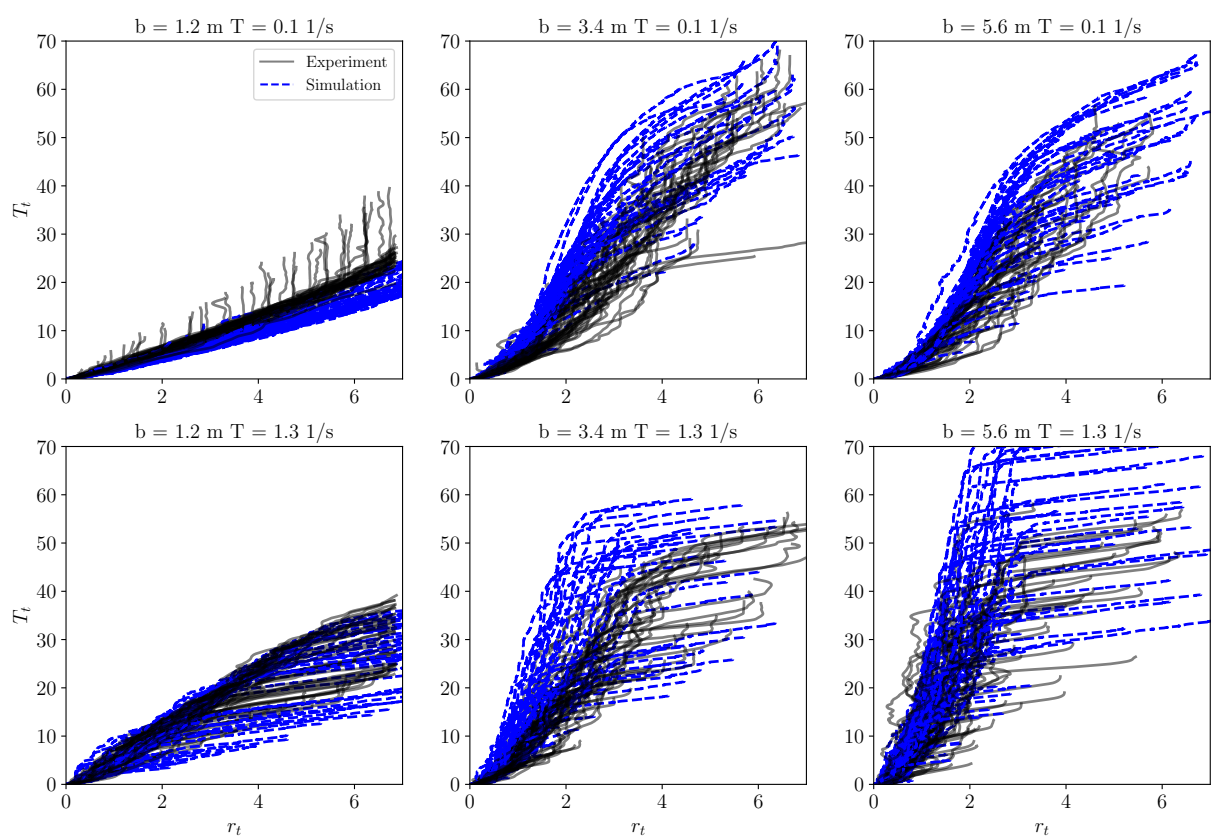

Figure 6 Time to target plots in respect to the target density for $b=\{1.2,3.4,5.6\} \mathrm{m}$ from left to right. The experimental data is shown in black and the simulations in blue. Top row shows high motivation while bottom row shows low motivation.

\section{Conclusion}

The speed-based model with simple interactions utilised here is surprisingly capable to reproduce observed emergent phenomena. The experimentally studied quantities can not only be reproduced qualitatively, but also quantitatively. The density increase, lane formation in narrow corridors and the relationship between the time to target $T_{t}$ and the distance to target $r_{t}$ show a good agreement between simulations and the experimental data. The model can distinguish between different motivations by varying only the slope parameter $T$ which leads to an increase in density, the disappearance of lanes in the corridor, and transitions between regimes of fast and slow contraction with an initial waiting period. This result has further implications for the interpretation of emergent phenomena in pedestrian flow. The experiments discussed were inspired by a preceding study that investigated a similar situation of different corridor geometries in front of a bottleneck [16]. In this study it was argued that certain aspects of human behaviour, like social norms, are needed to explain the observed behavior. Here we have shown that a simple model only containing volume exclusion and a slope factor depended collision avoidance combined with proper boundary conditions is capable of reproducing the experimental findings. 


\section{References}

[1] Chraibi, M., Tordeux, A., Schadschneider, A., Seyfried, A.: Modelling of pedestrian and evacuation dynamics. Encyclopedia of Complexity and Systems Science pp. 122 (2018)

[2] Chowdhury, D., Ludger, S., Schadschneider, A.: Statistical physics of vehicular traffic and some related systems. Physics Reports 329(4-6), 199-329 (2000). doi:10.1016/s0370-1573(99)00117-9

[3] Helbing, D.: Traffic and related self-driven many-particle systems. Reviews of Modern Physics 73(4), 1067-1141 (2001). doi:10.1103/revmodphys . 73.1067

[4] Schadschneider, A., Kirchner, A., Nishinari, K.: CA approach to collective phenomena in pedestrian dynamics. Lecture Notes in Computer Science Cellular Automata p. 239-248 (2002). doi:10.1007/3-540-45830-1_23

[5] Portz, A., Seyfried, A.: Analyzing stop-and-go waves by experiment and modeling. Pedestrian and Evacuation Dynamics p. 577-586 (2011). doi:10.1007/978-1-4419-9725-8_52

[6] Schadschneider, A., Klingsch, W., Klüpfel, H., Kretz, T., Rogsch, C., Seyfried, A.: Evacuation Dynamics: Empirical Results, Modeling and Applications, pp. 31423176. Springer, New York (2009). doi:10.1007/978-0-387-30440-3_187

[7] Helbing, D., Molnár, P., Farkas, I.J., Bolay, K.: Self-organizing pedestrian movement. Environment and planning B: planning and design 28(3), 361-383 (2001)

[8] Chraibi, M., Ezaki, T., Tordeux, A., Nishinari, K., Schadschneider, A., Seyfried, A.: Jamming transitions in force-based models for pedestrian dynamics. Physical Review E 92(4) (2015). doi:10.1103/physreve.92.042809

[9] Chraibi, M., Schadschneider, A., Seyfried, A.: On force-based modeling of pedestrian dynamics. Modeling, Simulation and Visual Analysis of Crowds The International Series in Video Computing p. 23-41 (2013). doi:10.1007/978-1-4614-8483-7_2

[10] Köster, G., Treml, F., Gödel, M.: Avoiding numerical pitfalls in social force models. Physical Review E 87(6) (2013). doi:10.1103/physreve.87.063305

[11] Chraibi, M., Kemloh, U., Schadschneider, A., Seyfried, A.: Force-based models of pedestrian dynamics. Networks and Heterogeneous Media 6(3), 425-442 (2011). doi:10.3934/nhm.2011.6.425

[12] Kretz, T.: On oscillations in the social force model. Physica A: Statistical Mechanics and its Applications 438, 272-285 (2015). doi:10.1016/j.physa.2015.07.002 
[13] Tordeux, A., Chraibi, M., Seyfried, A.: Collision-free speed model for pedestrian dynamics. In: Traffic and Granular Flow'15, pp. 225-232. Springer (2016)

[14] Hoogendoorn, S.P., Daamen, W.: Pedestrian behavior at bottlenecks. Transportation Science 39(2), 147-159 (2005)

[15] Seyfried, A., Passon, O., Steffen, B., Boltes, M., Rupprecht, T., Klingsch, W.: New insights into pedestrian flow through bottlenecks. Transportation Science 43(3), 395-406 (2009)

[16] Sieben, A., Schumann, J., Seyfried, A.: Collective phenomena in crowds-where pedestrian dynamics need social psychology. PLOS ONE 12(6), 1-19 (2017). doi:10.1371/journal.pone.0177328

[17] Adrian, J., Seyfried, A., Sieben, A.: Crowds in front of bottlenecks at entrances from the perspective of physics and social psychology. Journal of The Royal Society Interface 17(165), 20190871 (2020). doi:10.1098/rsif.2019.0871

[18] Daamen, W., Hoogendoorn, S.: Experimental research of pedestrian walking behavior. Transportation Research Record: Journal of the Transportation Research Board (1828), 20-30 (2003)

[19] Helbing, D., Buzna, L., Johansson, A., Werner, T.: Self-organized pedestrian crowd dynamics: Experiments, simulations, and design solutions. Transportation Science 39(1), 1-24 (2005)

[20] Garcimartín, A., Maza, D., Pastor, J.M., Parisi, D.R., Martín-Gómez, C., Zuriguel, I.: Redefining the role of obstacles in pedestrian evacuation. New Journal of Physics 20(12), 123025 (2018). doi:10.1088/1367-2630/aaf4ca

[21] Kretz, T., Grünebohm, A., Schreckenberg, M.: Experimental study of pedestrian flow through a bottleneck. Journal of Statistical Mechanics: Theory and Experiment 2006(10), P10014 (2006)

[22] Zuriguel, I., Echeverría, I., Maza, D., Hidalgo, R.C., Martín-Gómez, C., Garcimartín, A.: Contact forces and dynamics of pedestrians evacuating a room: The column effect. Safety Science 121, 394-402 (2020). doi:10.1016/j.ssci.2019.09.014

[23] Haghani, M., Sarvi, M., Shahhoseini, Z.: When 'push' does not come to 'shove': Revisiting 'faster is slower' in collective egress of human crowds. Transportation Research Part A: Policy and Practice 122, 51 (2019)

[24] Haghani, M.: Empirical methods in pedestrian, crowd and evacuation dynamics: Part ii. field methods and controversial topics. Safety Science 129, 104760 (2020). doi:10.1016/j.ssci.2020.104760 
[25] Feliciani, C., Nishinari, K.: Measurement of congestion and intrinsic risk in pedestrian crowds. Transportation Research Part C: Emerging Technologies 91, 124-155 (2018). doi:10.1016/j.trc.2018.03.027

[26] Fischer, M., Jankowiak, G., Wolfram, M.T.: Micro- and macroscopic modeling of crowding and pushing in corridors. Networks and Heterogeneous Media 15, 405 (2020). doi:10.3934/nhm.2020025

[27] Helbing, D., Farkas, I., Vicsek, T.: Simulating dynamical features of escape panic. Nature 407(6803), 487-490 (2000). doi : 10 . $1038 / 35035023$

[28] Tajima, Y., Takimoto, K., Nagatani, T.: Scaling of pedestrian channel flow with a bottleneck. Physica A: Statistical Mechanics and its Applications 294(1-2), 257-268 (2001)

[29] Nagatani, T.: Dynamical transition and scaling in a mean-field model of pedestrian flow at a bottleneck. Physica A: Statistical Mechanics and Its Applications 300(3-4), 558-566 (2001)

[30] Burstedde, C., Klauck, K., Schadschneider, A., Zittartz, J.: Simulation of pedestrian dynamics using a two-dimensional cellular automaton. Physica A: Statistical Mechanics and its Applications 295, 507-525 (2001). doi:10.1016/s0378-4371(01)00141-8

[31] Tang, M., Jia, H., Ran, B., Li, J.: Analysis of the pedestrian arching at bottleneck based on a bypassing behavior model. Physica A: Statistical Mechanics and its Applications 453, 242-258 (2016). doi:10.1016/j.physa.2016.02.044

[32] Parisi, D.R., Patterson, G.A.: Influence of bottleneck lengths and position on simulated pedestrian egress. Papers in Physics 9, 090001 (2017). doi:10.4279/pip.090001

[33] Haghani, M., Sarvi, M.: Simulating pedestrian flow through narrow exits. Physics Letters A 383(2-3), 110-120 (2019). doi:10.1016/j.physleta.2018.10.029

[34] Bandini, S., Crociani, L., Gorrini, A., Nishinari, K., Vizzari, G.: Unveiling the hidden dimension of pedestrian crowds: Introducing personal space and crowding into simulations. Fundamenta Informaticae 171(1-4), 19-38 (2019). doi:10.3233/fi-2020-1870

[35] Kirchner, A., Klüpfel, H., Nishinari, K., Schadschneider, A., Schreckenberg, M.: Simulation of competitive egress behavior: comparison with aircraft evacuation data. Physica A: Statistical Mechanics and its Applications 324(3-4), 689-697 (2003). doi:10.1016/s0378-4371(03)00076-1 
[36] Huang, L., Wong, S., Zhang, M., Shu, C.W., Lam, W.H.: Revisiting hughes' dynamic continuum model for pedestrian flow and the development of an efficient solution algorithm. Transportation Research Part B: Methodological 43(1), 127-141 (2009). doi:10.1016/j.trb.2008.06.003

[37] Graf, A.: Automated routing in pedestrian dynamics. Master Thesis (Fachhochschule Aachen, Campus Jülich) (2015)

[38] Buchmüller, S., Weidmann, U.: Parameters of pedestrians, pedestrian traffic and walking facilities. IVT Schriftenreihe 132 (2006). doi:10.3929/ethz-b-000047950

[39] Pedestrian data archive. https://ped.fz-juelich.de/da. Accessed: 2021-08-11

[40] Steffen, B., Seyfried, A.: Methods for measuring pedestrian density, flow, speed and direction with minimal scatter. Physica A: Statistical Mechanics and its Applications 389(9), 1902-1910 (2010) 